\title{
LOCAL IgA-MEMORY RESPONSE TO BACTERIAL ANTIGENS*
}

\author{
David F. Keren, Patricia J. Scott, Roderick A. McDonald, \\ and Scott E. Kern \\ Department of Pathology \\ The University of Michigan Medical Center \\ Ann Arbor, Michigan 48109
}

\section{INTRODUCTION}

For over 100 years, it has been known that oral immunization can protect against challenge by certain enteropathogenic bacteria. ${ }^{1-3}$ The mechanism of this protection was unclear as most individuals did not demonstrate strong serum agglutinins to the orally administered vaccine. Work by Davies in 1922 provided evidence that agglutinating antibodies in stool specimens from patients with dysentery correlated better with the disease activity than serum antibody levels. ${ }^{4}$ Following the demonstration by Tomasi et al. in 1965 that IgA was the main antibody on mucosal surfaces, a new area of investigation was opened that led to the elucidation of the mechanism of protection by oral vaccination. ${ }^{5}$

In order to follow the development of the local $\operatorname{IgA}$-immune response, we developed a chronically isolated ileal loop model in rabbits. ${ }^{6}$ This model allowed us to sample ileal loop secretions daily. In other studies, we immunized the isolated segment directly with bacteria, toxins, or other macromolecules.

In the present study, we have used this model to demonstrate that a mucosal IgA-memory response can be elicited following oral immunization with Shigella flexneri antigen.

\section{MATERIALS AND METHODS}

\section{Preparation of Chronically Isolated Ileal Loops}

The surgical creation of ileal, Thiry-Vella loops in rabbits has been described in detail previously. ${ }^{6}$ In brief, while three to four $\mathrm{kg}$ New Zealand White rabbits are anesthetized with xylazine and ketamine, a midline abdominal incision is made and the terminal ileum is identified. A $20 \mathrm{~cm}$ segment of ileum containing a grossly identifiable Peyer's patch is isolated with its vascular supply intact. Silastic tubing (Dow-Corning) is sewn into each end of the isolated segment. This tubing is then brought out through the midline incision and tunneled subcutaneously to the nape of the neck where it is exteriorized and secured. Intestinal continuity is restored by an end-to-end anastomosis, and the midline incision is closed in two layers.

Each day approximately two to four $\mathrm{ml}$ of secretions and mucous that collect in the isolated loops are expelled by injecting $20 \mathrm{ml}$ of air into one of the silastic tubes. The slightly opaque, colorless fluid and mucous expelled from the tubing is

\footnotetext{
*This work was supported in part by the U.S. Army Medical Research and Development Command Contract DAMD 1/-80-C-0113.
} 
studied for specific immunoglobulin content. A subsequent flush with $20 \mathrm{ml}$ of sterile saline helps to remove adherent mucous. This saline is then removed by repeated gentle flushes of air. With proper daily care, $80 \%-90 \%$ of rabbits can complete experiments lasting one to two months.

\section{Enzyme-Linked Immunosorbent Assay (ELISA)}

The ELISA procedure has been described in detail previously. ${ }^{7.8}$ Briefly, microtiter wells are coated with a solution containing Shigella flexneri lipopolysaccharide (Westphal preparation). Immediately prior to testing serum samples or loop secretions, the antigen solution is removed, and wells are washed with a phosphate buffer containing Tween 20 (PT). The fluid to be assayed is diluted in the PT buffer and incubated in the coated wells and in uncoated wells [to control for nonspecific adsorption) for four hours on horizontal rotary shaker. The plates are washed with PT and incubated with either alkaline phosphatase-conjugated goat anti-rabbit IgA or staphylococcal protein A overnight on the shaker. Following another PT wash, substrate reaction is carried out with nitrophenyl phosphate in carbonate buffer. The OD $405 \mathrm{~nm}$ of the substrate reaction is determined using a Titer Tek microELISA reader. Kinetics of the enzyme-substrate reaction are extrapolated to 100 minutes. The OD $405 \mathrm{~nm}$ of uncoated wells is subtracted from the OD $405 \mathrm{~nm}$ of coated wells. Specific IgG and IgA standards prepared as described previously ${ }^{7}$ are processed daily with the unknown fluids.

\section{Bacterial Preparations}

Three live Shigella strains, one acetone-killed strain, and one Westphal lipopolysaccharide preparation containing Shigella flexneri $2 a$ somatic antigens were used in these studies. Shigella flexneri strain M4243 was shown to invade the surface epithelium and to persist with ulcer formation. ${ }^{9}$ Shigella X16 is a hybrid of S. flexneri-Escherichia coli that invades surface epithelium, may form ulcers, but does not thrive (persist) in guinea pig intestine. ${ }^{10} \mathrm{~S}$. flexneri strain 2457-0 does not form ulcers and does not invade surface epithelium. ${ }^{11}$ The preparations of acetone-killed strain M4243 (AK-M4243) and the Shigella lipopolysaccharide preparations have been described previously. ${ }^{11-13}$

\section{Shedding of Live Shigella}

The presence of live bacteria in the stool and in loop secretions was assessed by culturing both stool samples and loop secretions on MacConkey agar plates as previously described. ${ }^{14}$

\section{Statistical Analysis}

Statistical analysis of the data was performed in the Midas computer of the Statistical Research Laboratory at The University of Michigan. The F-test result and significance were determined for each group. 


\section{RESULTS}

In earlier studies, we immunized the chronically isolated ileal loops directly with various antigen preparations. This immunization was done by injecting live or killed antigen preparations in $4 \mathrm{ml}$ of phosphate-buffered saline directly into the isolated loops. This material was left in the loops overnight and flushed out the next morning. Data from some of these earlier studies are summarized in FIGURE 1. In these studies, we found that chronically isolated ileal loops stimulated by various preparations of Shigella antigen contain considerable secretory IgA activity, but at most, trivial IgG activity against these antigens. ${ }^{13.14}$ Virtually no IgA and usually no or trivial IgG directed against Shigella flexneri antigens were found in sera from these rabbits.

When the isolated ileal loops were immunized directly with $10^{8}$ live bacteria, in doses given on days 0,7 , and 14 (day of surgery = day -1 ), there was no significant difference in the local IgA response for the three live antigen forms used (invasive M4243, invasive Shigella X16, noninvasive $2457-0$ ).${ }^{15}$ As shown in FIGURE 1, however, acetone-killed Shigella flexneri achieved a weaker local IgA response in the isolated ileal loop secretions. The lipopolysaccharide preparation

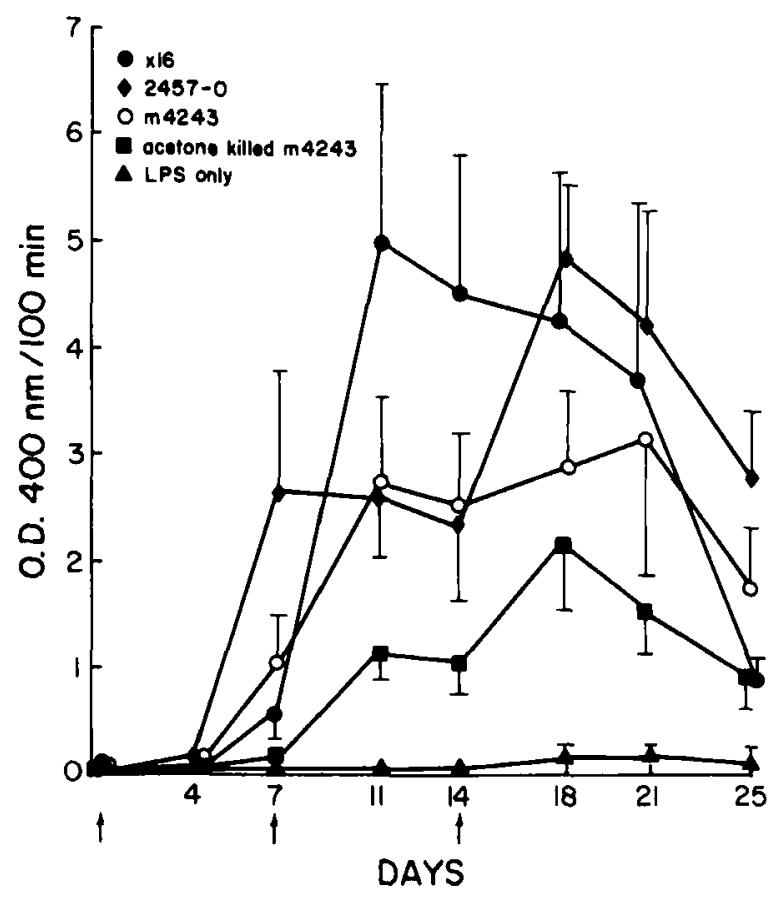

FIGURE 1. Mean IgA anti-shigella responses in ileal loop secretions from groups of rabbits immunized directly in the isolated loop with $10^{\circ}$ live M4243, X16, 2457-0, $2.5 \mathrm{mg}$ of acetone-killed M4243 per ml, or 100 ug of hot phenol-water prepared Shigella LPS. The vertical axis expresses the net optical density (O.D.) and the horizontal axis indicates days after first intraloop stimulation. Standard errors of mean (SEM) indicated. (D.F. Keren et al. ${ }^{15}$ With permission from Infection and Immunity). 


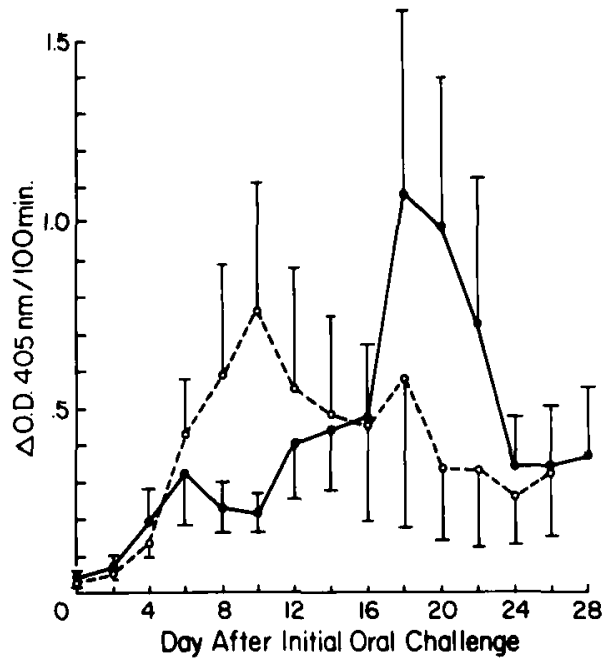

FIGURE 2. Mean IgA anti-shigella responses in ileal loop secretions from rabbits immunized orally with a single dose of $10^{10}$ live Shigella X16 on day $0(0)$ or from rabbits given $10^{10}$ live Shigella X16 on days 0,7 , and $14(\bullet)$. SEM indicated. (D.F. Keren. ${ }^{8}$ With permission from the Journal of Immunology.)

(given in a dose equivalent to the amount of lipopolysaccharide in $10^{8}$ bacteria) was ineffective in achieving a significant mucosal immune response. ${ }^{5}$

Whereas our chronically isolated ileal loop model in rabbits has been useful for following the kinetics of the local immune response to initial antigen stimulation, it has been difficult to use this model for studying the local IgA-anamnestic response of the bowel. First, it is difficult to maintain these isolated ileal loops for periods longer than one month. Animals that do survive for long periods of time (some have been kept alive with isolated ileal loops for as long as 10 months), usually require several subsequent surgical procedures to drain abscesses and correct abdominal problems. Further, some animals require antibiotic therapy for various related and unrelated infections. It is obvious that these are undesirable events in an experimental model to follow the immune response against infectious diseases. Second, by directly immunizing the isolated ileal loops, we are creating an extremely artificial situation. That is, if one were immunizing a population, or if an infection were occurring naturally, the vaccine or pathogen would pass through gastric acid, bile, pancreatic digestive enzymes, and normal intestinal flora and food products. Any or all of the aforementioned might alter the ability of the orally administered antigen to stimulate the mucosal immune response.

Therefore, in order to make our model system more relevant to both the natural infection and to a potential vaccine program for humans, we immunized the animals orally, rather than stimulating the isolated ileal loops directly. The local IgA response was followed by studying the secretions from the isolated ileal loops for specific IgA activity at various times following oral immunization. The isolated ileal loops are separated from the intestinal contents and, therefore, are never directly exposed to the antigen. 
This approach evolves from the theory that a common mucosal immune system exists. That such a system exists has been demonstrated by several laboratories. ${ }^{16-18}$ If this common mucosal immune system exists, it should be possible to create the isolated ileal loop, give an oral dose of antigen to that animal and follow the intestinal responses in the local secretions.

Indeed, following a single oral dose of $10^{10}$ live Shigella X16, the kinetics of the local IgA response in the chronically isolated ileal loop secretions paralleled the response seen when the loops were directly immunized with the same live antigen (FIGURE 2). ${ }^{8}$ No enhancement of this response was seen following three doses of live oral Shigella X16. When $10^{10}$ heat-killed Shigella X16 were given orally, similar, albeit weaker, local IgA responses were seen (FIGURE 3). ${ }^{8}$ In

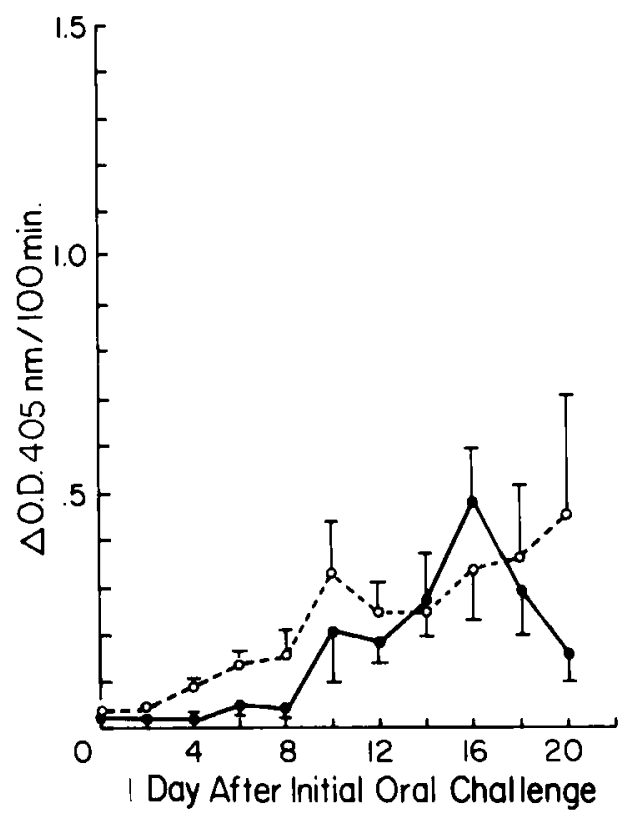

FIGURE 3. Mean IgA anti-shigella responses in ileal loop secretions from rabbits immunized orally with a single dose of $10^{10}$ heat-killed Shigella X16 on day $0(0)$ or from rabbits given $10^{10}$ heat-killed Shigella X16 on days 0,7 , and 14 (•). SEM indicated. (D.F. Keren. With permission from the Journal of Immunology.)

neither situation were there significant local IgG or serum IgG or IgA responses directed against these bacteria.

To determine whether a local IgA-memory response could be elicited by oral priming, a group of ten rabbits that did not have chronically isolated ileal loops was primed by giving three weekly, oral doses of $10^{10}$ live Shigella X16 to each rabbit. The animals were rested for two months after the third dose. Then, a chronically isolated ileal loop was surgically created in each animal and the animals were challenged with a single oral dose of $10^{10}$ live Shigella X16 on the day after surgery. 
TABLE 1

Local IgA-Memory Response in Rabit Ileal Loop Secretions after Oral Priming WITH LIVE SHIGELLA X16 ${ }^{8}$

\begin{tabular}{ccccc}
\hline \hline $\begin{array}{c}\text { Day After } \\
\text { Challenge* }\end{array}$ & Not Primed $\dagger$ & Live Shigella X16 $\neq$ & Significance $\S$ & F-STAT* $^{\star}$ \\
\hline 0 & $.047 \pm .006$ 1 & $.234 \pm .086$ & .0026 & 10.077 \\
1 & $.041 \pm .012$ & $.213 \pm .066$ & .0029 & .825 \\
2 & $.062 \pm .013$ & $.499 \pm .134$ & .0001 & 19.205 \\
3 & $.047 \pm .014$ & $.567 \pm .133$ & .0001 & 19.086 \\
4 & $.136 \pm .042$ & $1.778 \pm .438$ & .0001 & 27.790 \\
5 & $.218 \pm .103$ & $1.626 \pm .387$ & .0001 & 23.568 \\
6 & $.435 \pm .149$ & $1.610 \pm .298$ & .0007 & 13.173 \\
7 & $.519 \pm .513$ & $1.833 \pm .350$ & .0014 & 11.358 \\
8 & $.591 \pm .306$ & $1.363 \pm .248$ & .0151 & 6,322 \\
10 & $.532 \pm .210$ & $.979 \pm .249$ & N.S. & - \\
\hline \hline
\end{tabular}

*Day 0 - Day of final antigen challenge

† Animals given $10^{10}$ live Shigella X16 orally on day $0(n-19)$

$\ddagger$ Animals given $10^{10}$ live Shigella X16 orally on days $-75,-68,-61$ prior to oral challenge on day $0(n-10)$

§As determined by F-test

TResults expressed as mean optical density (O.D.) $405 \mathrm{~nm} / 100$ minutes \pm standard error of mean (S.E.M.) of IgA antibodies specific for Shigella antigen as determined by ELISA

$\|$ N.S. = not significant ( $>.02$ by F-test)

${ }^{\star \star}$ F-STAT $=$ F-test value

A striking local IgA-memory response was seen in these animals as compared to the nonprimed animals (TABLE 1). ${ }^{8}$ By contrast, the animals that received this same dosage schedule with killed Shigella X16 followed by a challenge with a single dose of killed Shigella, showed no evidence of a local IgA-memory response (TABLE 2$)^{B}$

TABLE 2

lack of IgA-Memory Response After Oral Priming with Killed Shiegella X16

\begin{tabular}{cccc}
\hline \hline $\begin{array}{c}\text { Day After } \\
\text { Challenge }\end{array}$ & Not Primed $\dagger$ & $\begin{array}{c}\text { Primed With } \\
\text { Killed Shigella X16 }\end{array}$ & Significance \\
\hline 0 & $.040 \pm .008 \uparrow$ & $.057 \pm .02$ & N.S.\| \\
2 & $.046 \pm .011$ & $.040 \pm .14$ & N.S. \\
4 & $.089 \pm .016$ & $.108 \pm .05$ & N.S. \\
6 & $.140 \pm .031$ & $.173 \pm .063$ & N.S. \\
8 & $.161 \pm .049$ & $.120 \pm .041$ & N.S. \\
\hline \hline
\end{tabular}

${ }^{\star}$ Day 0 = day of final antigen challenge

†Unprimed animals given $10^{10}$ killed Shigella X16 orally on day $0(n-12)$

$\ddagger$ Animals orally primed with $10^{10}$ killed Shigella X16 on days $-75,-68,-61$ prior to oral challenge on Day 0 ( $\mathrm{n}=11$ )

§Significance assessed by F-test

IResults expressed as mean optical density [O.D.] $405 \mathrm{~nm} / 100$ minutes \pm S.E.M. for Shigella antigen as determined by ELISA

$\|$ N.S. $=$ Not significant ( $>.02$ by F-test) 
During these studies, some rabbits were given erythromycin for pulmonary infections. All of the rabbits given the erythromycin developed poor local IgA-memory responses (FIgure 4). To see if this erythromycin effect could be repeated, a second group of rabbits was given the regimen for the memory studies with live Shigella X16 and then given erythromycin concurrently with the challenge. As shown in Figure 4, a significantly lower IgA-memory response was seen in the erythromycin-treated rabbits.

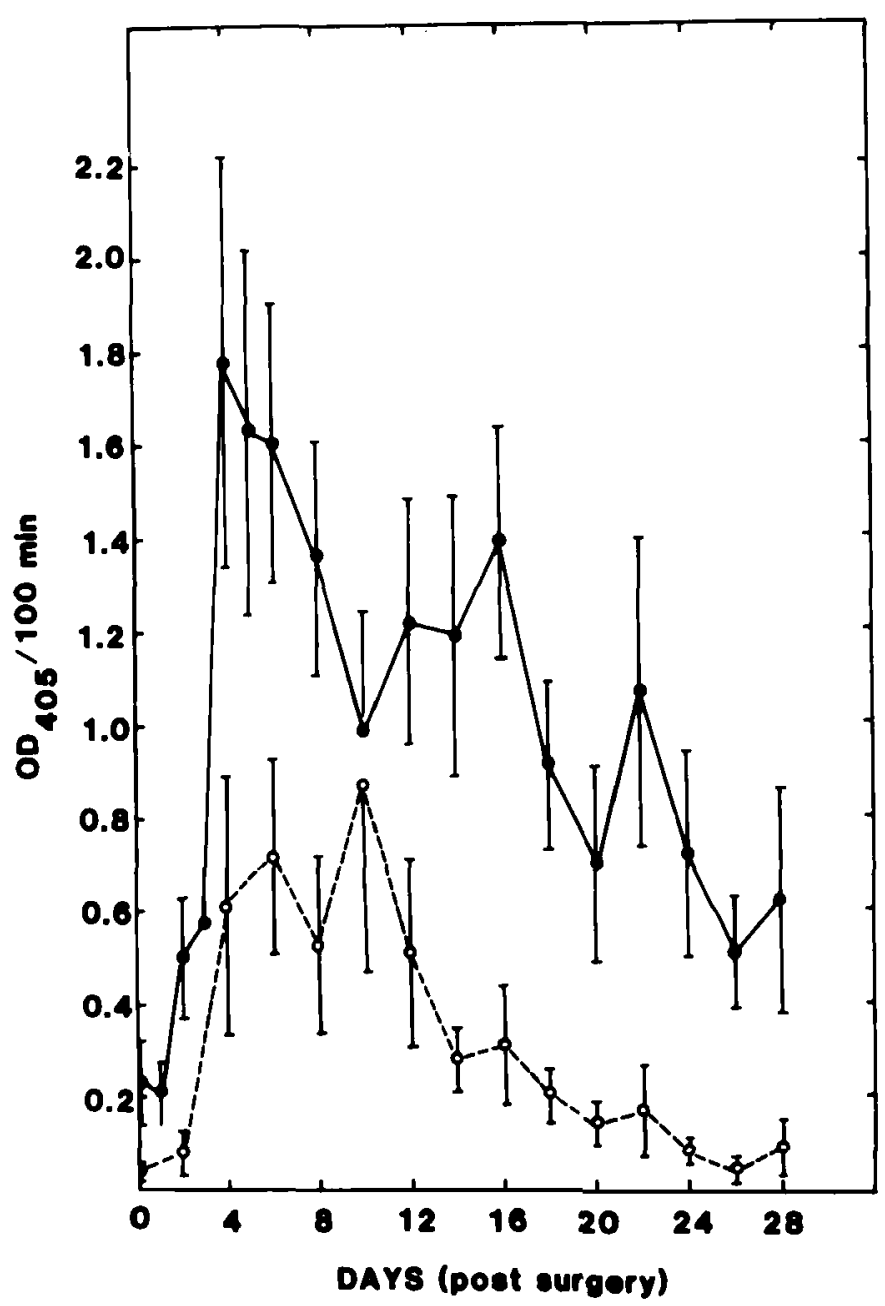

FIGURE 4. Mean IgA anti-shigella response in secretions from isolated ileal loops of rabbits primed with three oral doses of $10^{10}$ live shigella on days $-74,-67$, and -60 and challenged with a single oral dose on day 0 (one day after surgical creation of the isolated ileal loop.) One group of rabbits had no exposure to erythromycin (•); the other was given erythromycin for several days before and after the challenge dose of live Shigella X16 (이. SEM indicated. 


\section{Discussion}

In the present studies, we have used our chronically isolated ileal loop model in rabbits as a probe to follow the local IgA-memory response of the intestine after either oral or parenteral priming.

In our previous studies, we have shown that the intestinal secretions from chronically isolated ileal loops directly stimulated with various Shigella preparations will contain considerable secretory IgA but no, or only little, IgG directed against the Shigella. ${ }^{6-8,13-15}$ The lack of locally produced IgG is not due to rapid degradation of IgG as the isolated loops are separated from the proteolytic effects of gastric acid, bile, and the enzymes trypsin, pepsin, and chymotrypsin. ${ }^{19}$ Also, direct stimulation of the isolated loops by Shigella antigens has resulted in little or no systemic (serum) IgG against Shigella, unless the systemic immune response was previously primed by a subcutaneous or intravenous dose of Shigella. ${ }^{15}$

Although direct immunization of the chronically isolated ileal loop was useful for studying the kinetics of the local IgA response to Shigella antigens, it had major drawbacks. First, the effects of gastric acid, bile, and pancreatic digestive enzymes on either a natural infection or a potential oral vaccine were artifically bypassed by directly stimulating the chronically isolated ileal loops. Second, for studying the mucosal memory response, it was difficult to maintain chronically isolated loops for periods lasting longer than one to two months. Therefore, in the present study, rather than directly stimulating the isolated loops, we used the chronically isolated loops as a probe for following the local immune response. Because we were interested in studying the intestinal response, we chose an oral route of immunization. Pierce has demonstrated that the site of mucosal stimulation influences where the locally stimulated B-cells will lodge. ${ }^{20}$

These studies show that the kinetics of the IgA response to Shigella in our isolated ileal loops after a single oral immunization follow the same sequence as when the isolated loops were stimulated directly; this finding is further evidence for the common mucosal immune system. Further, it establishes this model system as a valid probe for events occurring locally in the intestine. We have then taken advantage of this system to determine whether a mucosal memory response to Shigella can be elicited by oral priming.

It is clear that we were able to achieve a mucosal memory response when live, but not killed, Shigella X16 were used to prime and challenge the rabbits orally.

The difference between the orally administered killed Shigella and live Shigella may be due to several factors. First, the live Shigella are able to multiply in the intestine. We found that stool from rabbits given oral Shigella would contain Shigella for as long as $\mathbf{1 0}$ days after each oral dose. No loop fluids were found to contain Shigella. This pattern of shedding is similar to that described for Shigella flexneri when they were administered orally to monkeys. ${ }^{11}$ Therefore, the actual dose of Shigella X16 for animals that received live oral Shigella was considerably greater than those that received killed Shigella. Second, Shigella $\mathrm{X} 16$ is a locally invasive strain. Actual invasion of the epithelium may be necessary for achieving or for enhancing the mucosal IgA-memory response. We do not think that the latter is the case, because preliminary studies in our laboratory using the noninvasive Shigella flexneri strain $2457-0$ have shown a mucosal IgA-memory response similar to that of the invasive Shigella X16. Last, the challenge antigen may be of importance. The challenge antigen for the rabbits primed with killed Shigella was heat-killed. It may be important to have a live bacteria for an effective challenge. This situation may be a dose-related phenomenon. 
Our studies using parenteral priming have shown no mucosal IgA-memory response in spite of the presence of systemic IgG against Shigella antigen.

Last, we record a peculiar effect of the antibiotic erythromycin on the local IgA-memory response of orally primed rabbits. When rabbits were treated with erythromycin at the time of their challenge dose, no local $\mathrm{IgA}$-memory response could be demonstrated in most of the rabbits. Only two of the ten rabbits studied showed evidence of an $\operatorname{IgA} A$-memory response. The mechanism of action of this effect is unclear at the present time. This effect could be of importance, however, in any vaccine program using live, attenuated bacteria.

In summary, the present studies offer our chronically isolated ileal loop model as a probe to follow the mucosal primary and memory responses to orally or parenterally administered Shigella antigens. We have shown that a significant local memory response is achievable by oral priming with live invasive, but not with heat-killed Shigella X16. Concurrent antibiotic administration may be able to alter the ability of animals to elicit such a mucosal memory response.

\section{ACKNOWLEDGMENT}

The authors thank Mrs. Mary Ann Byrnes for her excellent assistance in preparing this manuscript.

\section{REFERENCES}

1. Pasteur, L. 1880. Cited by Calmette, A. 1923. Les vaccinations microbiennes par voie buccale. Ann. Inst. Pasteur. 37: 900-920.

2. FERRAN, J. 1885. Cited by A. P. Fernandez. Vae Inventoribus Magnis! El doctor Ferran y el colera morbo asiatico en la guerra europea. Barcelona. Imp. La RenaixenzaXucla 13. 133-151. 1921.

3. BESREDKA, A. 1927. De la vaccination par voie buccale contre la dysenterie, la fievre typhoide et le cholera. Rev. d'Hyg. et de Med. Prev. 49: 445-463.

4. DAVIES, A. 1922. An investigation into the serological properties of dysenteric stool. Lancet. 2: 1009-1012.

5. Tomasi, T. B., E. M. Tan, A. Solomon \& R. A. Prendergast. 1965. Characteristics of an immune system common to certain external secretions. J. Exp. Med. 121: 101-124.

6. Keren, D. F., H. L. ElliotT, G. D. BROWN \& J. H. YARDLEY, 1975. Atrophy of villi with hypertrophy and hyperplasia of Paneth cells in isolated (Thiry-Vella) ileal loops in rabbits. Gastroenterology 68: 83-93.

7. KEREN, D. F. 1979. Enzyme-linked immunosorbent assay for immunoglobulin $G$ and immunoglobulin A antibodies to Shigella flexneri antigens. Infect. Immun. 24: 441-448.

8. Keren, D. F., S. E. Kern D. H. Bauer, P. J. Scott \& P. Porter. 1982. Direct demonstrtation in intestinal secretions of an IgA memory response to orally administered Shigella flexneri antigens. J. Immunol. 128: 475-479.

9. Formal, S. B., T. H. Kent, S. Austin \& E. H. LaBrec. 1966. Fluorescent antibody and histological study of vaccinated and control monkeys challenged with Shigella flexneri. J. Bacteriol. 19: 2368-2376.

10. Formal, S. B., E. H. LaBrec, T. H. Kent \& S. Falkow. 1965. Abortive intestinal infection with an Escherichia coli-Shigella flexneri hydrid strain. J. Bacteriol. 89: 1374-1382.

11. Formal, S. B., E. H. LaBrec, A. Palmer \& S. Falkow. 1965. Protection of monkeys against experimental shigellosis with attenuated vaccines. J. Bacteriol. 90: 63-68.

12. Formal, S. B., R. M. Maenza, S. Austin, \& E. H. LaBrec. 1967. Failure of parenteral 
vaccines to protect monkeys against experimental shigellosis. Proc. Soc. Exp. Biol. Med. 125: 347-349.

13. Keren, D. F., P. S. Holt, H. H. Collins, P. Gemski \& S. B. Formal. 1978. The role of Peyer's patches in the local immune response of rabbit ileum to live bacteria. J. Immunol. 120: 1892-1896.

14. Keren, D. F., P. S. Holt, H. H. Collins, P. Gemski \& S. B. Formal. 1980. Variables affecting the local immune response in Thiry-Vella loops. I. Role of immunization schedule, bacterial flora, and postsurgical inflammation. Infect. Immun. 28: 950956.

15. Keren, D. F., H. H. Collins, P. Gemski, P. S. Holt \& S. B. Formal. 1981. Role of antigen form in development of mucosal IgA response to Shigella flexneri antigens. Infect. Immun. 31: 1193-1202.

16. Cebra, J. J., R. Kamat, P. Gearhart, S. M. Robertson \& J. Tseng. 1977. The secretory IgA system of the gut. In Immunology of the gut. CIBA Foundation Symposium 46: 5-28.

17. McWilliams, M., J. M. Phillips-Quagliata \& M. E. Lamm. 1977. Mesenteric lymph node $B$ lymphoblasts which home to the small intestine are precommitted to IgA synthesis. J. Exp. Med. 145: 866-875.

18. MCDERMOTT, M.R. \& J. BIENENSTOCK. 1979. Evidence for a common mucosal immunologic system. I. Migration of B immunoblasts into intestinal, respiratory, and genital tissues. J. Immunol. 122: 1892-1897.

19. KEREN, D. F., P. J. SCOTT \& D. BAUER, 1981. Variables affecting the local immune response in Thiry-Vella loops. II. Stability of antigen-specific IgG and secretory IgA in acute and chronic Thiry-Vella loops. J. Immunol. 124: 2620-2624.

20. Pierce, N. F. \& W. C. Cray, JR. 1982. Determinants of the localization, magnitude, and duration of a specific mucosal IgA plasma cell response in enterically immunized rats. J. Immunol. 128: 1311-1315.

\section{Discussion of THE PAPER}

A. J. HusBand (The University of Newcastle, Newcastle, Australia): We know that IgA is intimately bound up with intestinal mucus. Did you handle your samples in any special way to accommodate for the mucus production that changes in isolated loops?

D. F. KEREN (The University of Michigan, Ann Arbor, Mich.): We handle all our specimens in the same way. We immediately centrifuge them and remove the mucus that might have some IgA bound to it. We assay the overlying clear supernatants that we obtain from these.

HusBanD: You showed that you had IgA antibody produced in isolated, nonimmunized loops when you immunized the intact intestine.

KEREN: That is correct.

HusBand: And do you propose that this is a counterpart of the common mucosal immune system?

KEREN: Yes.

HUSBAND: I would suggest that the cells that are migrating to those loops are in transit. In the absence of antigen in those loops, they would not be induced to proliferate and persist. Does your data support my explanation of that result, that the IgA-antibody levels in these loops are more transient or lower in magnitude than a loop that is directly immunized?

KEREN: It seems improbable, because the antibody that we see does seem to 
persist for at least three to four weeks, albeit, at relatively lower levels. One would think that if antibody were just passing through the loops, that it would not persist quite that long.

HUSBAND: Does that antibody level, however, compare favorably with antibody levels in directly immunized loops?

KEREN: No. The antibody in the directly immunized loops was much higher. 